\title{
Identifying the nature of surface chemical modification for directed self-assembly of block copolymers
}

\author{
Laura Evangelio ${ }^{1,2}$, Federico Gramazio ${ }^{2}$, Matteo Lorenzoni ${ }^{1}$, Michaela Gorgoi ${ }^{3}$, \\ Francisco Miguel Espinosa4 ${ }^{4}$, Ricardo García ${ }^{4}$, Francesc Pérez-Murano*1 \\ and Jordi Fraxedas ${ }^{*}$
}

\section{Full Research Paper}

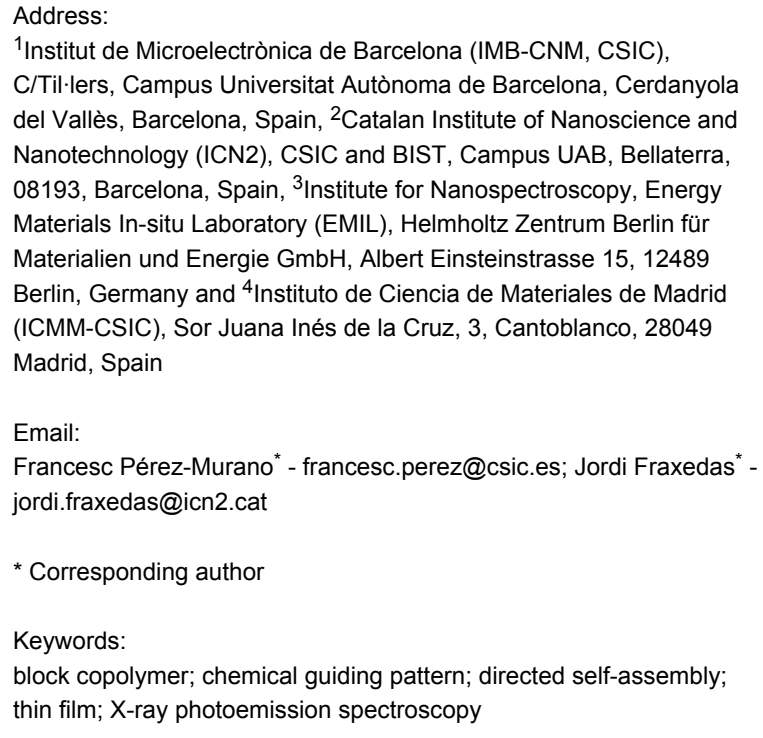

${ }^{1}$ Institut de Microelectrònica de Barcelona (IMB-CNM, CSIC), $\mathrm{C} / \mathrm{Til} \cdot$ lers, Campus Universitat Autònoma de Barcelona, Cerdanyola del Vallès, Barcelona, Spain, ${ }^{2}$ Catalan Institute of Nanoscience and Nanotechnology (ICN2), CSIC and BIST, Campus UAB, Bellaterra, 08193, Barcelona, Spain, ${ }^{3}$ Institute for Nanospectroscopy, Energy Materials In-situ Laboratory (EMIL), Helmholtz Zentrum Berlin für Materialien und Energie $\mathrm{GmbH}$, Albert Einsteinstrasse 15, 12489 Berlin, Germany and ${ }^{4}$ Instituto de Ciencia de Materiales de Madrid (ICMM-CSIC), Sor Juana Inés de la Cruz, 3, Cantoblanco, 28049 Madrid, Spain

Email:

Francesc Pérez-Murano* - francesc.perez@csic.es; Jordi Fraxedas* jordi.fraxedas@icn2.cat

* Corresponding author

Keywords:

block copolymer; chemical guiding pattern; directed self-assembly; thin film; X-ray photoemission spectroscopy

Beilstein J. Nanotechnol. 2017, 8, 1972-1981. doi:10.3762/bjnano.8.198

Received: 07 June 2017

Accepted: 31 August 2017

Published: 21 September 2017

Associate Editor: S. R. Cohen

(C) 2017 Evangelio et al.; licensee Beilstein-Institut. License and terms: see end of document.

\begin{abstract}
In recent years, block copolymer lithography has emerged as a viable alternative technology for advanced lithography. In chemicalepitaxy-directed self-assembly, the interfacial energy between the substrate and each block copolymer domain plays a key role on the final ordering. Here, we focus on the experimental characterization of the chemical interactions that occur at the interface built between different chemical guiding patterns and the domains of the block copolymers. We have chosen hard X-ray high kinetic energy photoelectron spectroscopy as an exploration technique because it provides information on the electronic structure of buried interfaces. The outcome of the characterization sheds light onto key aspects of directed self-assembly: grafted brush layer, chemical pattern creation and brush/block co-polymer interface.
\end{abstract}

\section{Introduction}

Directed self-assembly (DSA) of block copolymers (BCPs) is a chemical-based complementary alternative to traditional patterning methods providing sub-10 nm resolution, low-cost processing and high throughput [1-3]. Moreover, it is one of the most promising techniques for the development of the next gen- eration of nanoelectronic devices and circuits, as it is compatible with current manufacturing processes.

BCPs are macromolecules derived from more than one species of monomers with inter-monomer covalent bonding. Due to the 
repulsion between different blocks, the BCPs tend to segregate and undergo a separation phase with controllable dimensions and functionalities due to unfavorable enthalpic interactions [4] The global parameters that govern the phase behavior of BCPs are given by the $\chi \cdot N$ product, where $\chi$ stands for the Flory Huggins parameter and $N$ the number of statistical segments in a BCP chain, which is related to the free energy of the system and the composition of the blocks $[5,6]$. When the BCP selfassembly is used in combination with surface prepatterning, aligned structures of alternative phases of the blocks can be obtained. This is the principle of DSA. The main advantages are a relaxation of the resolution requirements of traditional lithography methods, as the period of the prepattern can be larger than the final period of the self-assembled pattern, and an improvement in both line-edge and line-width roughness.

There are mainly two techniques to direct the self-assembly of BCPs: graphoepitaxy and chemical epitaxy. In graphoepitaxy, the BCPs are aligned by a topographical substrate pattern[7-9], whereas in chemical epitaxy the self-assembly is driven by the difference of surface free-energies between the domains of the copolymer and the chemical prepattern[2,10-12]. Currently, the industry is more focused on chemical epitaxy rather than on graphoepitaxy due to the fact that the BCP is guided in with negligible changes in the height step of the patterns and because of its easier integration[13].

In chemical epitaxy DSA, the interfacial energies between each domain of the copolymer and the chemically patterned surfaces strongly influence the final morphology and micro-domain ordering. Therefore, an accurate control of the surface chemistry is needed, for example, to obtain the desirable orientation during self-assembly (parallel or perpendicular lamellae or cylinders), to avoid dewetting phenomena or to minimize the presence of defects. Generally, in chemical epitaxy DSA, the background (unmodified) surface should be slightly attractive to one of the domains of the copolymers while the chemically modified areas should be slightly attractive to the other one.

In order to understand the resulting $\mathrm{BCP}$ morphology when it is self-assembled on the top of a chemical guiding pattern, it is important to determine which chemical interactions occur between both modified and unmodified regions of the substrate with each block of the copolymer. One technique especially suited for the characterization of buried interfaces is hard X-ray high kinetic energy photoelectron spectroscopy (HAXPES) [14]. Photoemission is a well-known technique which provides information on the electronic structure of surfaces. Its high surface sensitivity arises from the small mean free path of the outcoming photoelectrons in solid matter. Using conventional excitation sources, kinetic energies below $1500 \mathrm{eV}$ can be achieved, which correspond approximately to a $2 \mathrm{~nm}$ probing depth for inorganic materials. The possibility of acquiring photoemission spectra at higher kinetic energies, as high as $10 \mathrm{keV}$, has permitted the exploration of the chemical environment of subsurface regions down to more than $20 \mathrm{~nm}$ for polymeric materials [15]. HAXPES reaches its full potential when using synchrotron radiation as an excitation source since, in this case, photon energy (and thus kinetic energy) can be tuned so that the probing depth can be also varied in a controlled and continuous manner. Nevertheless, one drawback of this technique, in particular when using polymers and organic materials, is the irreversible damage caused by the impinging beam. Therefore, this is a matter that has to be carefully addressed in any measurement, even for inorganic materials[16,17].

Here, we have used HAXPES as well as conventional XPS to investigate the chemical changes that occur during the processing steps involved in DSA. As it is depicted in Figure 1, three chemical epitaxy DSA processes have been investigated in order to determine the dominant interactions between the substrate (a brush layer covering a silicon wafer, left in the Figure) and the block copolymer domains. The first DSA (two steps) process uses electron beam lithography (EBL) [12] on a poly(methyl methacrylate) (PMMA) resist with a subsequent substrate functionalization with oxygen plasma of the uncovered areas (top of Figure 1). The two other processes are based on direct writing (one-step) methods, thus avoiding the use of a resist. The selected methods are EBL (middle in Figure 1) and parallel oxidation nanolithography [18] (PON) (bottom of the Figure), respectively. The PON method is performed by contacting a conductive mold with the brush surface while applying a voltage under high humidity conditions. Details on the preparation methods can be found in the Experimental section.

\section{Results and Discussion Two-step electron beam and oxygen plasma modification}

Figure 2 shows SEM images of directed self-assembled films of PS- $b$-PMMA BCP prepared on substrates modified with EBL and oxygen plasma and annealed using the two selected annealing processes described above: (Figure 2a) $230{ }^{\circ} \mathrm{C}$ in nitrogen atmosphere for $5 \mathrm{~min}$ and cooling in nitrogen and (Figure $2 \mathrm{~b}$ ) $260^{\circ} \mathrm{C}$ in nitrogen atmosphere for $5 \mathrm{~min}$ and cooling in air. A scheme with the same scale of the distribution of the generated patterns is shown in the right of the figure as a guide. Before imaging, the PMMA blocks were removed by exposing the sample to $50 \mathrm{sccm}$ of oxygen flow at $500 \mathrm{~W}$ for $18 \mathrm{~s}$ in order to visualize the efficiency of the DSA process. From the figure, it becomes evident that when the brush cooling is performed in nitrogen rather than in air, the polymer has 

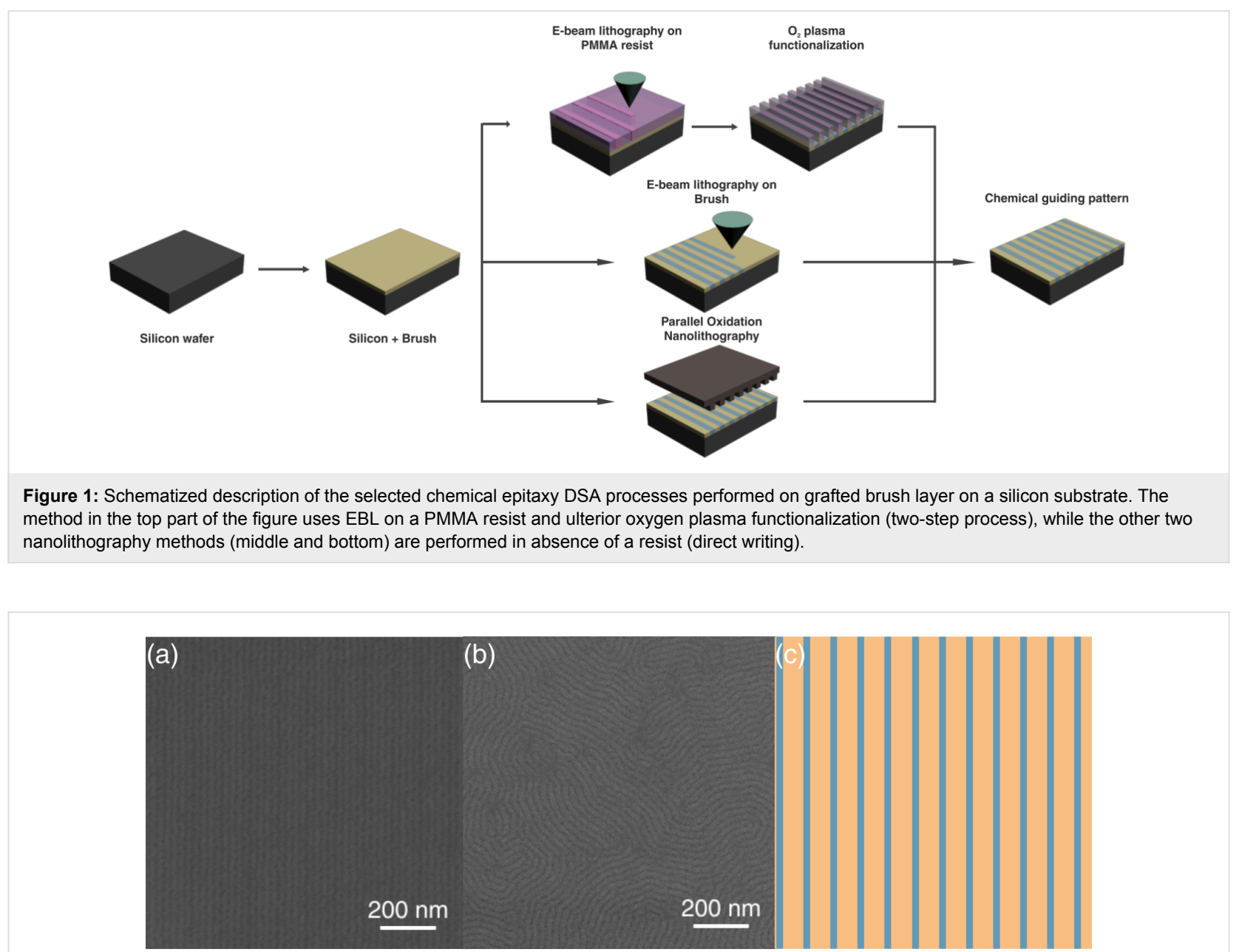

Figure 2: SEM images of PS-b-PMMA BCP after removal of the PMMA blocks prepared after EBL and oxygen plasma functionalization of grafted PS-OH layers deposited on silicon wafers (see Figure 1) after (a) annealing at $230^{\circ} \mathrm{C}$ and cooling in nitrogen and (b) annealing at $260{ }^{\circ} \mathrm{C}$ and cooling in air. (c) Scheme of the induced patterns (same scale as in (a) and (b)).

the proper surface free energy to induce the alignment of the $\mathrm{BCP}$ after the lithography and BCP spin-coating.

In order to understand the origin of the influence of the DSA process, we have performed HAXPES experiments on PS-OH grafted layers cooled under the selected conditions. Figure $3 \mathrm{a}$ shows the experimental HAXPES C 1s line (continuous black line) of the sample cooled in nitrogen together with a leastsquare fit after background subtraction (see caption of figure for details). The most prominent line (continuous red line), with a binding energy of $285.1 \mathrm{eV}$ corresponds to $\mathrm{C}-\mathrm{C}$ and $\mathrm{C}-\mathrm{H}$ bonding. The continuous blue line in Figure $3 \mathrm{a}$, with a binding energy of $286.2 \mathrm{eV}$, corresponds to the hydroxyl bonding of the PS-OH. The $\pi-\pi^{*}$ shake-up feature at $291.9 \mathrm{eV}$, characteristic of a pure PS spectrum (continuous magenta line) [19], is also observed. Figure $3 \mathrm{~b}$ compares the zoomed in spectra of the $\mathrm{C} 1 \mathrm{~s}$ lines corresponding to the sample cooled in air (orange) and that cooled in nitrogen (black), respectively. The figure evidences a small but clear increase in intensity of the region corresponding to hydroxyl bonding for the sample cooled in air. We point out that this minor effect in the $\mathrm{C} 1 \mathrm{~s}$ line can only be observed because of the high-energy resolution used in the HAXPES experiments and that parallel XPS measurements of samples prepared under the same conditions did not show any significant difference. The higher density of hydroxyl bonding induces higher attraction to PMMA blocks due to the affinity with carbonyl PMMA groups. In this case, the chemical guiding patterns created afterwards on the sample cooled in air will not be effective since the brush is already slightly PMMA affine before the oxygen plasma functionalization. Conversely, when the sample is cooled down in nitrogen, PS does not undergo oxidation. As a consequence, such a sample is slightly affine to PS before functionalization. When chemical guiding stripes are defined on this substrate by oxygen plasma exposure (see Figure 2), there will be enough chemical contrast to guide the alignment of the BCP. We thus conclude that such a small 

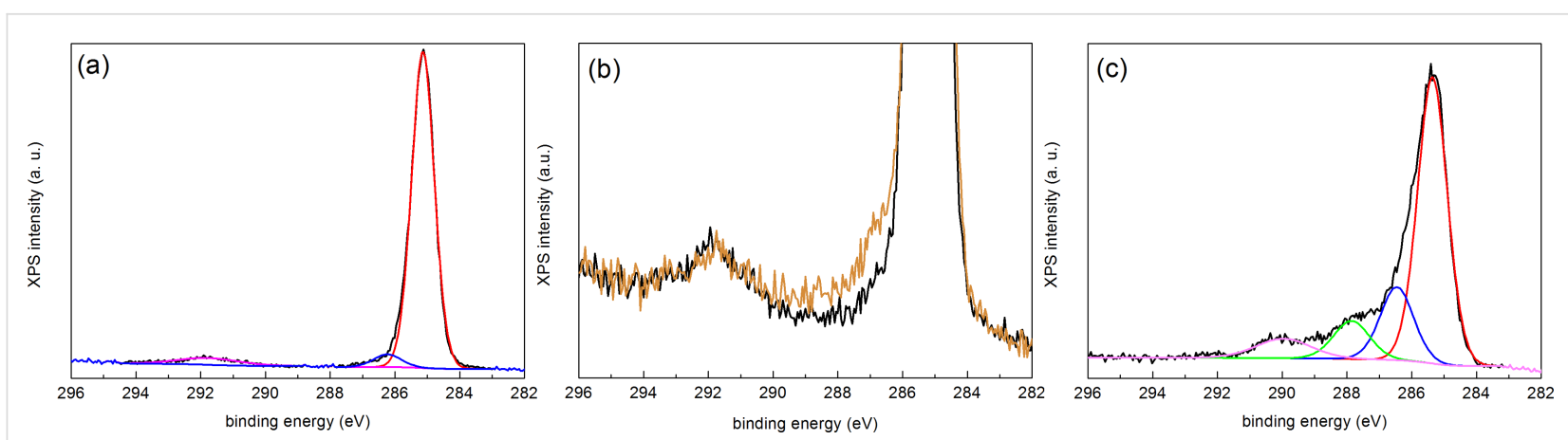

Figure 3: HAXPES spectra corresponding to the $\mathrm{C}$ 1s region of grafted PS-OH samples (a) cooled in nitrogen, (b) cooled in air (orange) and in nitrogen (black) and (c) cooled in nitrogen and exposed to oxygen plasma, taken with $2020 \mathrm{eV}$ using a SCIENTA R4000 hemispherical analyser. Least-square fits of the experimental data (continuous black line) after background subtraction (Shirley-type) are shown in (a) and (c) using a combination of Gaussian $(70 \%)$ and Lorentzian $(30 \%)$ functions under the constraint of identical full width at half maximum (FWHM) values for all components.

increase in hydroxyl bonding is sufficient to disable the alignment capabilities of the PS-OH brush layer (see Figure 2a,b).

Once the favourable cooling conditions for the preparation of the polymer brush layer were analysed, we investigated the effect of the functionalization upon exposure to oxygen plasma. Figure $3 \mathrm{c}$ shows the $\mathrm{C} 1 \mathrm{~s}$ HAXPES spectrum taken with $2020 \mathrm{eV}$ photons of the sample cooled in nitrogen after the oxygen plasma treatment. The comparison with Figure 3a evidences an increase in intensity towards higher binding energies in the $\approx 286-291 \mathrm{eV}$ region, which corresponds to contributions from different carbon-oxygen bonding configurations, as a result of the effect of the oxygen plasma exposure on the PS-OH brush layer. The continuous red and blue lines, with binding energies of 285.3 and $286.5 \mathrm{eV}$, respectively, exhibit a $0.2-0.3 \mathrm{eV}$ shift towards higher energies as compared to Figure $3 \mathrm{a}$, indicating different charging. The continuous blue line, corresponding to hydroxyl bonding, becomes more intense as compared to Figure 3a. Two new features are observed at 287.9 and $290 \mathrm{eV}$ binding energies, which are assigned to the carbonyl $(\mathrm{C}-\mathrm{O}$, continuous green line $)$ and carboxyl $(\mathrm{O}-\mathrm{C}=\mathrm{O}$, continuous pink line) contributions, respectively. Thus, oxygen plasma activates the brush layer surface by creating a distribution of $\mathrm{C}-\mathrm{O}$ bonding, while annealing and cooling in air induces essentially hydroxylation of the surface. Thus, the combination between optimal process conditions for grafting the polymer brush layer and an adequate chemical functionalization by oxygen plasma exposure leads to the possibility to generate efficient chemical patterns for guiding the selfassembly of the BCP.

Additional information can be obtained from AFM experiments. Figure 4a,b shows the AFM topography and phase images, respectively, of the chemical guiding patterns [20]. In both
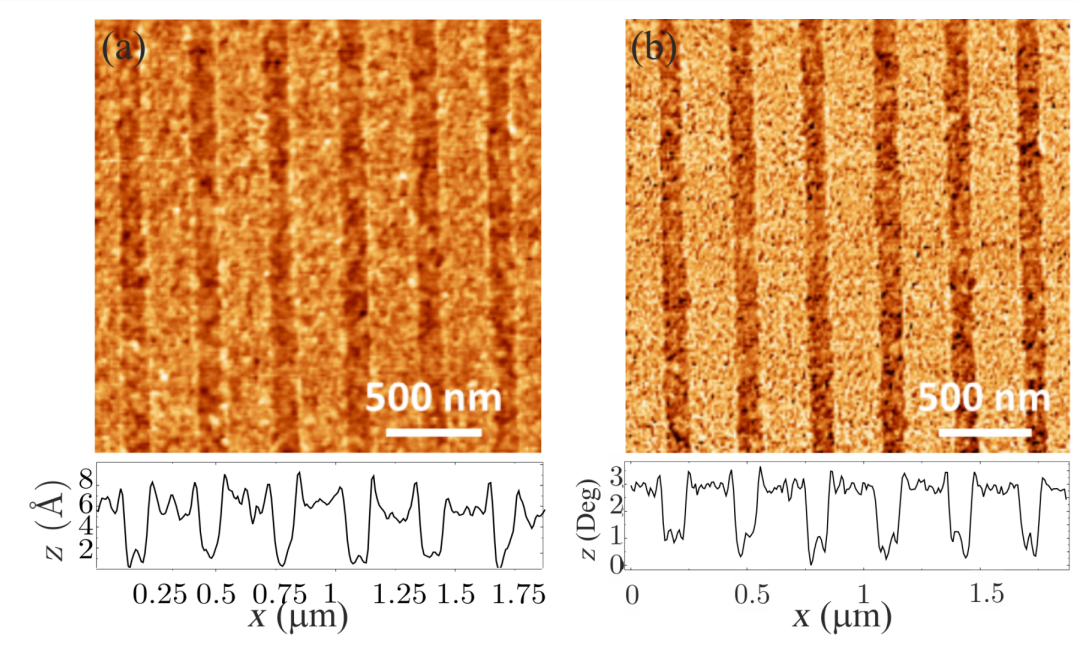

Figure 4: AFM topography (a) and phase (b) images of a chemical guiding pattern created by EBL followed by oxygen plasma modification on the brush sample cooled in nitrogen (see Figure 1). Representative cross sections are shown below both images. 
images the brighter lines correspond to the unexposed PS-OH brush layer, while the darker lines stand for the modified surface. The chemical contrast revealed by the AFM phase image is a signature of the fact that the unexposed PS-OH stripes are slightly affine to PS while the stripes exposed to oxygen plasma are slightly affine to PMMA due to the oxidation of the polymer. Applying the same method to create the guiding patterns on the sample cooled in air resulted in a surface which did not show any contrast when performing the AFM phase characterization. Furthermore, the oxygen plasma exposure induces a removal of about $0.4 \mathrm{~nm}$ of PS-OH, as deduced from the topography image in Figure 4a. This is in line with complementary X-ray reflectometry (XRR) results performed on annealed brush layers which deliver a brush thickness for unexposed and exposed surfaces of $4.6 \mathrm{~nm}$ and $3.9 \mathrm{~nm}$, respectively. The presence of some topography between the stripes may enhance the guiding efficiency of the chemical patterns [7-9], but such generated corrugation is not enough to induce the alignment of the BCP. This is confirmed by the results shown in Figure 2a,b.

Finally, we investigate the interfacial affinity between the PMMA block and both modified (oxygen plasma) and unmodified brush layers. For this matter $\approx 20 \mathrm{~nm}$ thick PMMA films have been deposited on top of grafted brush layers that have been unexposed and exposed to oxygen plasma, respectively, without prior EBL modification. Figure $5 \mathrm{a}$, b shows the $\mathrm{C} 1 \mathrm{~s}$ HAXPES spectra acquired with $3000 \mathrm{eV}$ photons together with the deconvolution using least-square fits after background subtraction. Both spectra show the characteristic $287.5 \mathrm{eV}$ (continuous green line) and $290 \mathrm{eV}$ (continuous magenta line) peaks of PMMA, corresponding to $\mathrm{O}-\mathrm{CH}_{3}$ and $\mathrm{O}-\mathrm{C}=\mathrm{O}$ configurations, respectively, with a 1:1 stoichiometric relationship [21]. The continuous red and blue lines correspond to $\mathrm{C}-\mathrm{C} / \mathrm{C}-\mathrm{H}$ bonding and to hydroxyl bonding, respectively, as described in Figure 3. The nominal $\mathrm{O}-\mathrm{C}=\mathrm{O}$ contribution to the full photoemission spectrum in pure PMMA is $20 \%$ (one carbon over the total five carbons in the monomer). Such proportion measured with XPS and HAXPES from PMMA films directly deposited on silicon substrates (no brush layers) is about $17-18 \%$, as obtained by comparing the area of the $\mathrm{O}-\mathrm{C}=\mathrm{O}$ contribution to the total area of the $\mathrm{C} 1 \mathrm{~s}$ line. The lower proportion can be ascribed to contamination during exposure to air. From Figure 5a,b we observe that the proportions are about $7 \%$ and $13 \%$ for the unexposed and exposed samples, respectively. Apart from surface contamination, the lower values are due to the contribution from the underlying $\mathrm{PS}-\mathrm{OH}$ brush layer, which adds to the main $\mathrm{C}-\mathrm{C} / \mathrm{C}-\mathrm{H}$ line, and to a lesser extent, to the $\mathrm{C}-\mathrm{OH}$ line. The lower the values, the larger the contribution from the brush layer, so that we can conclude that the unmodified brush layer is not uniformly covered by the relatively thick PMMA film as a result of the lower affinity between both materials (inefficient
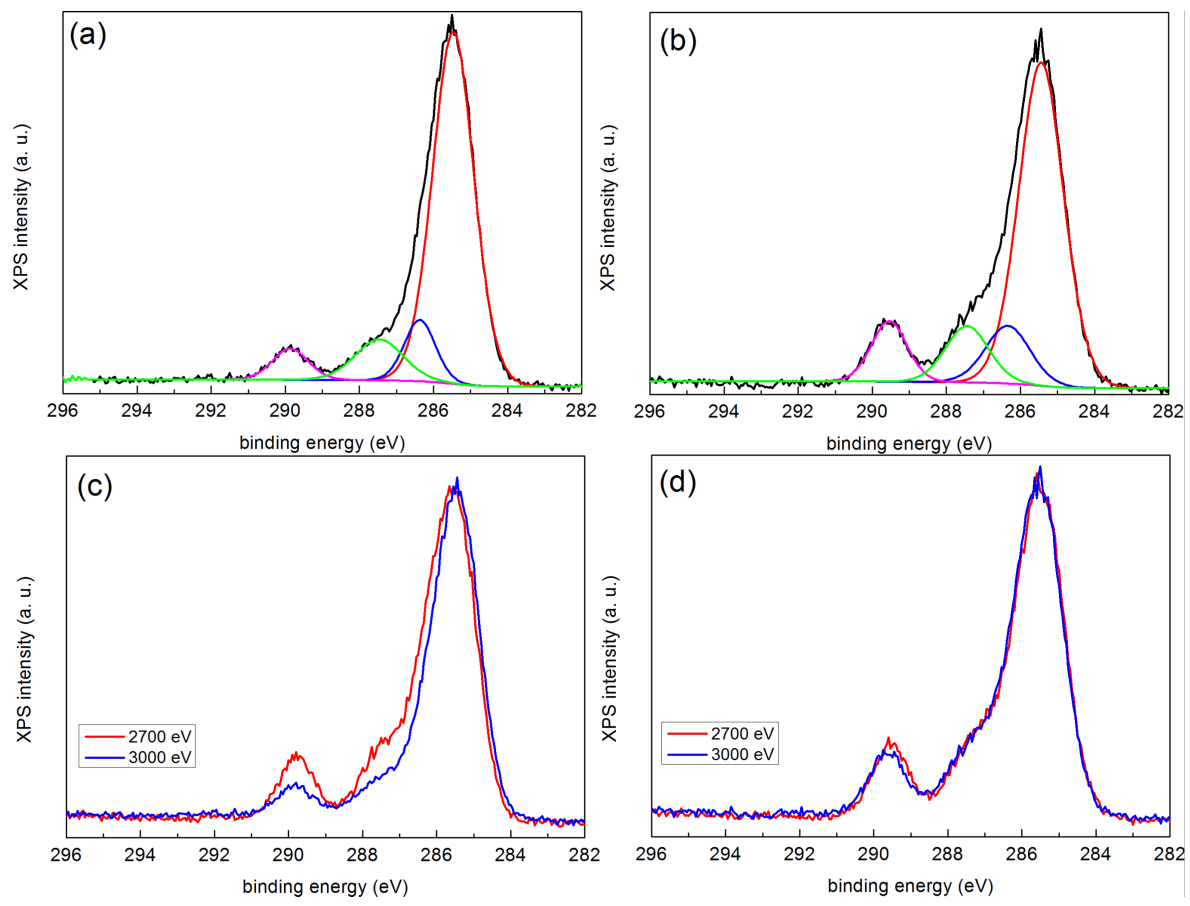

Figure 5: HAXPES spectra corresponding to the $\mathrm{C}$ 1s region of (a) unmodified and (b) modified samples, respectively, taken with 3000 eV photons including a deconvolution of the components using a least-square fit after background subtraction. Comparison of spectra taken with 2700 and 3000 eV photons of the unmodified (c) and modified (d) samples, respectively. Spectra in (c) and (d) have been normalized and aligned to the peak maxima. 
wetting). However, in the case of the sample exposed to oxygen plasma, the modified character of the brush layer triggers a higher affinity to PMMA (more efficient although non complete wetting).

This is further confirmed when the C 1s HAXPES spectra are taken at different photon energies, as shown in Figure $5 \mathrm{c}, \mathrm{d}$, where the spectra have been acquired at 2700 and $3000 \mathrm{eV}$, respectively. Increasing photon energy implies increasing kinetic energy and thus increasing probing depth. In the case of the unexposed sample, the mentioned proportion varies from $12 \%$ at $2700 \mathrm{eV}$ to $7 \%$ at $3000 \mathrm{eV}$ (5\% decrease) and the modified brush layer from $15 \%$ at $2700 \mathrm{eV}$ to $13 \%$ at $3000 \mathrm{eV} \mathrm{(2 \%} \mathrm{de-}$ crease). The calculated variations of the relative contribution of the $\mathrm{O}-\mathrm{C}=\mathrm{O}$ configuration between 2700 and $3000 \mathrm{eV}$ is less than $1 \%$ using a two-layer model for a $20 \mathrm{~nm}$ PMMA film homogeneously covering a $5 \mathrm{~nm}$ thick brush layer (see Supporting Information File 1, Figure S1). Thus, the larger decrease can be ascribed to the increasing contribution of the incompletely covered PS-OH substrate.

\section{Direct writing}

We discuss here the results obtained with resistless lithography methods, namely EBL [12] and PON [18]. Figure 6a shows a comparison between C 1s XPS spectra of three PS-OH brush layer treated surfaces after annealing at $230{ }^{\circ} \mathrm{C}$ and cooling in nitrogen (continuous red line), after EBL (continuous blue line) and with a freshly cleaved highly-oriented pyrolytic graphite (HOPG) surface (discontinuous black line). The surface modified by EBL shows a relatively large broadening and a strong shift towards lower binding energies, as compared to the sample modified by EBL and oxygen plasma. Binding energies have been referenced to the $\mathrm{Si} 2 \mathrm{p}_{3 / 2}$ peak $(99.3 \mathrm{eV})$ from the buried silicon substrate. The mentioned shift towards lower binding energies denotes the increasing presence of $\mathrm{sp}^{2}$ bonding based on the comparison with the results from a freshly cleaved HOPG sample (discontinuous black line), which shows a narrow peak centered at $284.4 \mathrm{eV}$, characteristic of $\mathrm{sp}^{2}$ bonding. Such increase in $\mathrm{sp}^{2}$ bonding is in line, although not a direct proof, with the cross-linking of PS due to electron beam exposure, as has been reported in the literature [22,23]. This contributes to the alignment of the BCP, as shown in Figure $6 \mathrm{~b}$.

Now, we focus the analysis on the sample prepared by PON. Figure 7a shows an AFM image of a chemical guiding pattern created by PON. The effect of the brush modification is an effective replication of the DVD pattern with modified regions (brighter in the AFM topography) slightly elevated $(1.1 \mathrm{~nm})$. The pitch of the DVD pattern is too large to achieve an aligned block/copolymer pattern. We used a DVD stamp in order to get a large area chemical guiding pattern useful for the HAXPES characterization. We also succeed in creating chemical guiding patterns with stamps of smaller pitch (see Supporting Information File 1, Figure S2), but in this case, we observed that the areas between lines were also chemically modified, preventing a block co-polymer chemical alignment. In any case, it was demonstrated in [11] that chemical patterns performed by local oxidation are very effective to align the block co-polymers if their geometrical dimensions are properly defined. Figure $7 \mathrm{~b}$ shows the Si 1s spectra taken at different photon energies in the 2020-3000 eV range. At $2020 \mathrm{eV}$ (black continuous line) only one feature is observed at about $1844 \mathrm{eV}$ binding energy. At higher photon energies, two more lines are identified at about 1841 and $1846 \mathrm{eV}$ binding energies, respectively, that become increasingly dominant for increasing photon energies. The 1841 and $1846 \mathrm{eV}$ features correspond to the buried $\mathrm{Si} / \mathrm{SiO}_{2}$ inter-
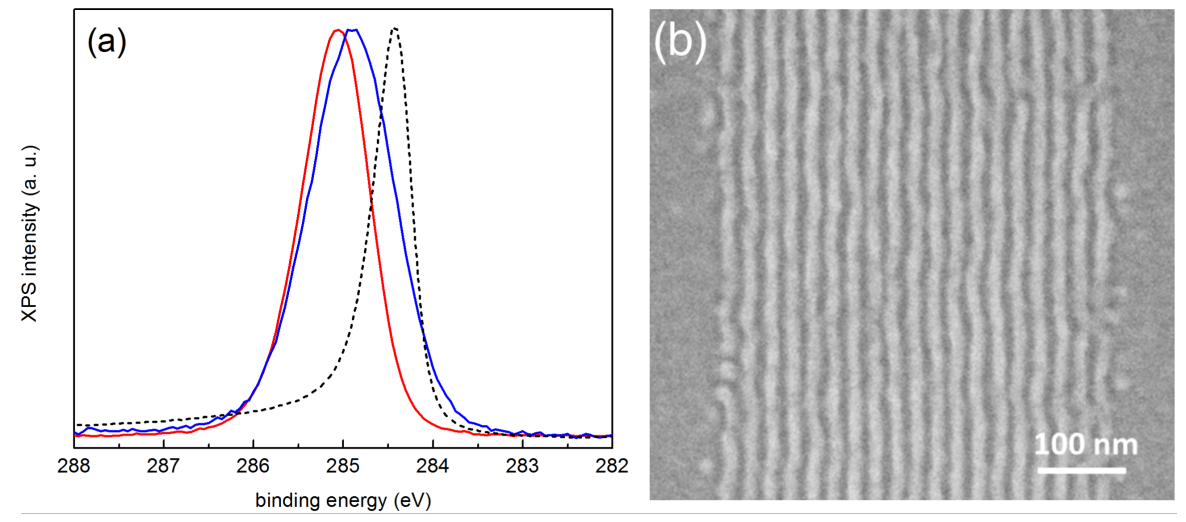

Figure 6: (a) C 1s XPS spectra of a grafted brush PS-OH layer after annealing at $230{ }^{\circ} \mathrm{C}$ and cooling in nitrogen (continuous red line), a sample modified by EBL (continuous blue line), and HOPG (discontinuous black line) using a PHOIBOS150 analyzer and monochromatic 1486.6 eV excitation. (b) SEM image of a $22 \mathrm{~nm}$ pitch PS-b-PMMA aligned in a pattern created by direct electron beam exposure. 


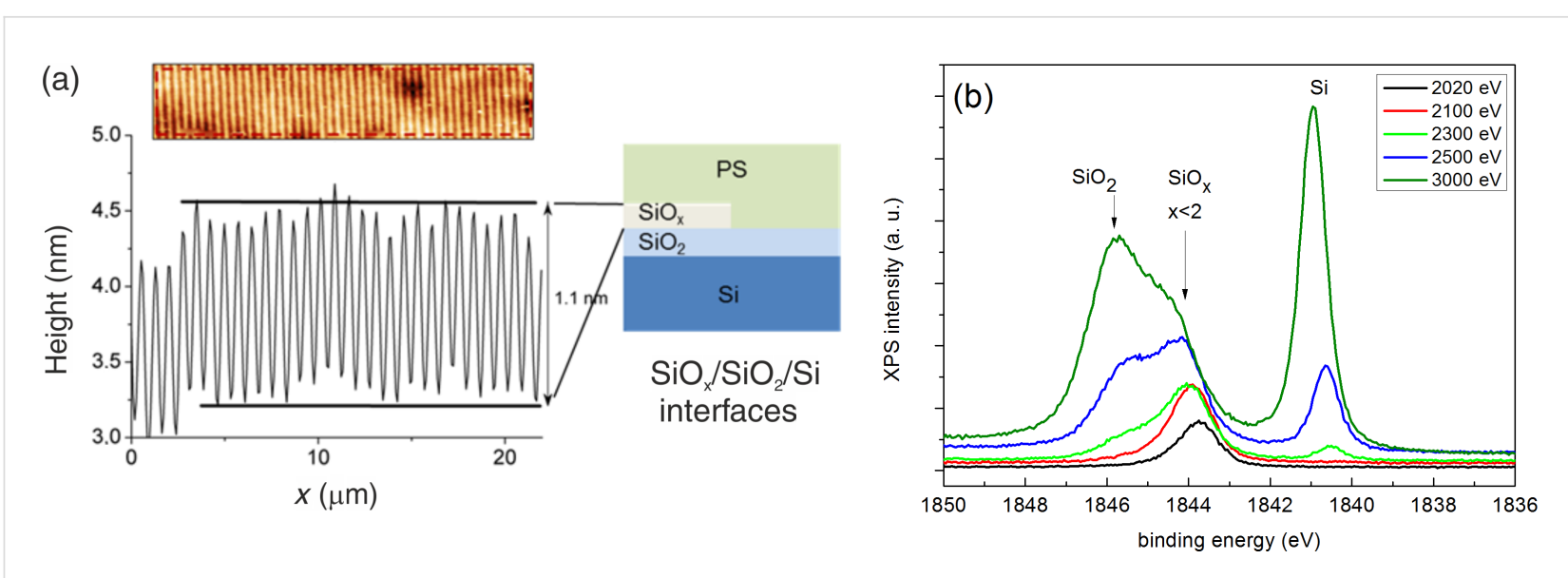

Figure 7: (a) AFM topography image and profile of the PS-OH brush annealed and cooled down in nitrogen after parallel oxidation nanolithography. A scheme is included showing the different regions. (b) HAXPES spectra of the Si 1s region at different photon energies.

face. Previous photoemission measurements performed at lower energy resolution using the Si $2 p$ line conclude that most of the oxide grown using PON is purely stoichiometric, although contribution from Si lower oxidation states may be present [24]. In references $[18,24,25]$ the $\mathrm{Si} 2 \mathrm{p}$ spin-orbit splitting is not resolved (compare to Figure S3 in Supporting Information File 1), which can preclude the observation of additional features in the region corresponding to the oxide.

In our case, we have selected the Si 1s line instead because of the higher interfacial sensitivity with photons above $2020 \mathrm{eV}$ as compared to the Si $2 p$ line. At $2020 \mathrm{eV}$, the corresponding kinetic energies are about $180 \mathrm{eV}$ and $1920 \mathrm{eV}$ for Si 1s and Si $2 p$, respectively, and the corresponding inelastic mean free path for electrons are $0.7 \mathrm{~nm}(\mathrm{Si})$ and $0.9 \mathrm{~nm}\left(\mathrm{SiO}_{2}\right)$ at $180 \mathrm{eV}$ and $4.1 \mathrm{~nm}(\mathrm{Si})$ and $4.9 \mathrm{~nm}\left(\mathrm{SiO}_{2}\right)$ at $1920 \mathrm{eV}$, respectively [26]. Thus, we can continuously follow the emergence of the three features as a function of the increasing probing depth from the surface with the Si 1s lines. In the case of the Si $2 p$ line, photon energies above $200 \mathrm{eV}$ should be used, which could not be achieved at the KMC-1 beamline.

We can thus conclude that the $1844 \mathrm{eV}$ line emerges from a region located on top of the $\mathrm{Si} / \mathrm{SiO}_{2}$ interface, as schematized in Figure $7 \mathrm{a}$, since it is the first feature that appears at the lower photon energy used. In addition, since the feature at $1844 \mathrm{eV}$ exhibits a lower binding energy as compared to the $1846 \mathrm{eV}$ counterpart, it can be assigned to a substoichiometric oxide layer $\left(\mathrm{SiO}_{x}\right)$, with partially oxidized silicon. Both lines are shifted due to charging induced by the grown oxide layers [25]. No relevant changes are observed in the $\mathrm{C} 1 \mathrm{~s}$ spectra taken at the same photon energies (apart from charging), although they do not correspond to the same probing depths, since the associated kinetic energies are above $1735 \mathrm{eV}$ for $\mathrm{C} 1 \mathrm{~s} \mathrm{eV}$, with inelastic mean free paths above $3.8 \mathrm{~nm}$ (see Supporting Information File 1, Figure S4).

It is worth mentioning that the use of brush layers of different thickness would produce a different phenomenology. For example, it is known that the use of brush layers thicker than $5 \mathrm{~nm}$ inhibits the interaction of the block copolymer with the substrate [27] and that for thinner layers, the surface neutralization provided by the brush layers is highly dependent on the polarity of the underlying substrate [28].

\section{Conclusion}

We have demonstrated that HAXPES using synchrotron radiation is a powerful spectroscopic tool to explore the chemical properties of surfaces and buried interfaces of brush layers for directed self-assembly of block copolymers, since valuable chemical information as a function of probing depth can be obtained.

The choice of the correct cooling of annealed PS-OH brush layers is of paramount importance in order to obtain an optimal DSA process. HAXPES characterization shows an increase in intensity in the energy range corresponding to hydroxyl bonds when the brush is cooled down in the presence of oxygen. This is consistent with the change in the chemical affinity of the brush layer with the BCP experimentally observed in DSA.

With regard to the functionalization of the PS-OH brush layers, it has been proven that oxygen plasma exposure activates the brush layers by generating diverse carbon-oxygen bonding which promotes higher affinity to PMMA blocks. Electron beam exposure increases $\mathrm{sp}^{2}$ bonding, promoting higher affinity to PS blocks that might be explained by cross-linking of PS. In 
the case of parallel oxidation nanolithography, HAXPES provides experimental evidence of the existence of a substoichiometric oxide between the brush layer and the $\mathrm{SiO}_{2} / \mathrm{Si}$ substrate.

\section{Experimental Preparation and chemical modification of brush layers}

The starting substrates were $<100>$ silicon wafers (p-type silicon of $4-40 \Omega \cdot \mathrm{cm}$ resistivity) with a native silicon oxide layer on top. A thin film of hydroxyl-terminated polystyrene (PS-OH, $M_{\mathrm{n}}=4.5 \mathrm{~kg} / \mathrm{mol}$ and PDI $=1.09$, purchased from Polymer Source), was deposited by spin-coating and annealed on the silicon substrate. Two different annealing conditions were used: (i) $260{ }^{\circ} \mathrm{C}$ in nitrogen atmosphere for $5 \mathrm{~min}$ and cooling in air and (ii) $230{ }^{\circ} \mathrm{C}$ in nitrogen atmosphere for $5 \mathrm{~min}$ and cooling in nitrogen.

The first DSA process is based on using EBL with a subsequent substrate functionalization with oxygen plasma exposure in order to chemically modify the brush layer and thus make it slightly affine to the other BCP domain (see top of Figure 1). In a first step, the brush is grafted on top of the activated silicon substrate and it is annealed. Then, the non-grafted brush layer is rinsed away by dipping the sample into toluene for $5 \mathrm{~min}$ in an ultrasound bath. Consequently, the EBL is performed on an $80 \mathrm{~nm}$ thick PMMA resist and after the development, the sample is briefly exposed to oxygen plasma in order to chemically modify the exposed areas and thus change their chemical affinity.

The two other processes are based on direct writing methods, thus avoiding the use of a resist. In the first case, the sample was exposed directly to an electron beam which modifies the chemical affinity of the PS-OH brush layer (see middle of Figure 1) [12]. The third method (PON) [18], shown in the bottom of Figure 1, is performed by contacting a conductive mold with the PS-OH surface while applying a voltage under conditions of high humidity. The stamp consists of a $1 \mathrm{~cm}^{2}$ piece of a DVD replica made with PDMS and coated with $100 \mathrm{~nm}$ gold film evaporated in high vacuum. The stamp surface presents parallel hillocks $320 \mathrm{~nm}$ wide and spaced $740 \mathrm{~nm}$. The height of the protrusions is $40 \mathrm{~nm}$. To transfer the patterns from the stamp to the substrate a 35-40 V bias voltage (sample positive) for a time ranging between 40 and $180 \mathrm{~s}$ was applied while the stamp was gently $(50 \mathrm{kPa})$ pressed upon the substrate. Relative humidity was kept above $70 \%$. These parameters are similar to the parameters used to perform an oxidation scanning probe lithography (SPL) experiment [29]. The authors have already demonstrated the efficiency of oxidation SPL to create chemical guiding patterns for DSA [11].
After defining the chemical guiding patterns, lamellar poly(styrene- $b$-methyl methacrylate) (PS- $b$-PMMA) dissolved in a $1.15 \%(\mathrm{w} / \mathrm{w})$ toluene solution was spin-coated onto the substrates and annealed at $200{ }^{\circ} \mathrm{C}$ for $20 \mathrm{~min}$ in air. The obtained films exhibit a thickness of about $36 \mathrm{~nm}$ with the used conditions $(2750 \mathrm{rpm}$ for $60 \mathrm{~s})$. In addition, and in order to characterize the interface between the oxygen modified and unmodified substrates with PMMA domains, $\approx 20 \mathrm{~nm}$ thick PMMA $\left(M_{\mathrm{n}}=30 \mathrm{~kg} / \mathrm{mol}\right)$ layers were deposited by spin-coating on top of them.

\section{Characterization techniques}

The HAXPES experiments were performed at the HIKE endstation located at the KMC-1 beamline at the BESSY II synchrotron of the Helmholtz Zentrum Berlin für Materialien und Energie in Berlin (Germany) [30,31]. Monochromatic radiation in the 2020-6000 eV photon energy range was used in our experiments, impinging the sample surface at grazing incidence. Photo-emitted electrons were collected with a SCIENTA R4000 high-resolution hemispherical analyzer at near normal emission, with an upper limit in kinetic energy of 10,000 eV. Experiments were performed in an ultrahigh vacuum chamber with a base pressure in the high $10^{-9}$ mbar range. To prevent beam damage, measurements were taken at different locations on the sample. In addition, the radiation was stopped (beam shutter closed) when spectra were not acquired (e.g. in case of monochromator setting change, change of sample position, etc.). Ex situ XPS experiments were performed at room temperature with a SPECS PHOIBOS 150 hemispherical analyzer using monochromatic $\mathrm{A} 1 \mathrm{~K} \alpha(1486.6 \mathrm{eV})$ radiation as an excitation source at a base pressure in the $10^{-9}$ mbar range.

As compared to previously reported XPS experiments performed with conventional sources [32], it is important to note that: (i) our results have been obtained with high energy resolution (see Supporting Information File 1, Figure S1), (ii) no external charge neutralization source has been used to compensate for charging effects due to the insulating character of the polymers and (iii) the binding energies have been referenced to the $\mathrm{Si} 2 \mathrm{p}_{3 / 2}$ line of the underlying pure silicon substrate, with a binding energy of $99.3 \mathrm{eV}$ [33], as independently determined by XPS and corresponding to a moderately p-doped sample as well as to the Si $2 p_{3 / 2}$ line of a clean silicon reference sample [34]. Shifts induced by recoil effects [35,36] and band bending [37] are not considered here since we are interested in the relative position of the photoemission lines (rigid shits) rather than on their absolute binding energies.

The SEM images shown in this work have been obtained with an AURIGA system from Zeiss and a Dimension Icon atomic force microscopy (AFM) from Bruker. The film thickness was 
determined by means of X-ray reflectometry (XRR) performed using a Philips X'Pert Pro MRD diffractometer equipped with a parabolic mirror using $\mathrm{Cu} \mathrm{K} \alpha$ radiation (1.54187 ̊).

\section{Supporting Information}

Simulated proportion of the $\mathrm{O}-\mathrm{C}=\mathrm{O}$ contribution to the normalized $\mathrm{C} 1 \mathrm{~s}$ spectrum as a function of the photoelectron kinetic energy. AFM images of a mold oxidation stamp fabricated on glass by NIL and metalized $(5 \mathrm{~nm} \mathrm{Cr} / 70 \mathrm{~nm} \mathrm{Au})$ and corresponding parallel oxidation pattern on PS-OH. Photoemission spectra of the Si $2 p$ line taken with $2020 \mathrm{eV}$ photons of a $\mathrm{PS} / \mathrm{SiO}_{2} / \mathrm{Si}$ sample. Photoemission spectra of the $\mathrm{C} 1 \mathrm{~s}$ lines of the PON sample taken with 2020 and $3000 \mathrm{eV}$ photons.

\section{Supporting Information File 1}

Additional experimental results.

[http://www.beilstein-journals.org/bjnano/content/ supplementary/2190-4286-8-198-S1.pdf]

\section{Acknowledgements}

The research leading to these results has received funding from the European Community's Seventh Framework Programme (FP7/2007-2013) within the projects CoLiSA (FP7-ICT-201311) and SNM (FP7-ICT-2011-8) and from MINECO (Nanointegra, TEC2015-69864-R). Financial support under the CALIPSO Programme (HZBPHOTONS_CALIPSO-96) is highly appreciated. ICN2 acknowledges support from the Severo Ochoa Program (MINECO, Grant SEV-2013-0295) and from the CERCA Programme (Generalitat de Catalunya). Ricardo García acknowledges financial support from MAT2013-4458-R. Laura Evangelio acknowledges the Ministry of Education of Spain for her FPU grant (FPU 13/03746). We thank HZB for the allocation of synchrotron radiation beam time. Thanks are due to Dr. Ruslan Ovsyannikov for technical assistance. All polymeric materials have been supplied by Arkema.

\section{References}

1. Mansky, P.; Liu, Y.; Huang, E.; Russell, T. P.; Hawker, C. Science 1997, 275, 1458-1460. doi:10.1126/science.275.5305.1458

2. Ruiz, R.; Kang, H.; Detcheverry, F. A.; Dobisz, E.; Kercher, D. S.; Albrecht, T. R.; de Pablo, J. J.; Nealey, P. F. Science 2008, 321 , 936-939. doi:10.1126/science.1157626

3. Suh, H. S.; Kim, D. H.; Moni, P.; Xiong, S.; Ocola, L. E.; Zaluzec, N. J.; Gleason, K. K.; Nealey, P. F. Nat. Nanotechnol. 2017, 12, 575-581. doi:10.1038/nnano.2017.34

4. Seul, M.; Andelman, D. Science 1995, 267, 476-483. doi:10.1126/science.267.5197.476
5. Fasolka, M. J.; Mayes, A. M. Annu. Rev. Mater. Res. 2001, 31, 323-355. doi:10.1146/annurev.matsci.31.1.323

6. Farrell, R. A.; Fitzgerald, T. G.; Borah, D.; Holmes, J. D.; Morris, M. A. Int. J. Mol. Sci. 2009, 10, 3671-3712. doi:10.3390/ijms10093671

7. Segalman, R. A.; Yokoyama, H.; Kramer, E. J. Adv. Mater. 2001, 13, 1152-1155. doi:10.1002/1521-4095(200108)13:15<1152::AID-ADMA1152>3.0.CO; 2-5

8. Park, S.; Lee, D. H.; Xu, J.; Kim, B.; Hong, S. W.; Jeong, U.; Xu, T.; Russell, T. P. Science 2009, 323, 1030-1033. doi:10.1126/science. 1168108

9. Tiron, R.; Chevalier, X.; Gaugiran, S.; Pradelles, J.; Fontaine, H.; Couderc, C.; Pain, L.; Navarro, C.; Chevolleau, T.; Cunge, G.; Delalande, M.; Fleury, G.; Hadziioannou, G. Proc. SPIE 2012, 8323, 83230O. doi:10.1117/12.916400

10. Liu, C.-C.; Han, E.; Onses, M. S.; Thode, C. J.; Ji, S.; Gopalan, P.; Nealey, P. F. Macromolecules 2011, 44, 1876-1885. doi:10.1021/ma102856t

11. Fernández-Regúlez, M.; Evangelio, L.; Lorenzoni, M.; Fraxedas, J.; Pérez-Murano, F. ACS Appl. Mater. Interfaces 2014, 6, 21596-21602. doi:10.1021/am506600m

12. Evangelio, L.; Fernández-Regúlez, M.; Borrisé, X.; Lorenzoni, M.; Fraxedas, J.; Pérez-Murano, F. J. Micro/Nanolithogr., MEMS, MOEMS 2015, 14, 33511. doi:10.1117/1.JMM.14.3.033511

13. Lin, Y.; Chen, X., Eds. Advanced Nano Deposition Methods; Wiley-VCH: Weinheim, Germany, 2016. doi:10.1002/9783527696406

14. Drube, W. J. Electron Spectrosc. Relat. Phenom. 2013, 190, 125-314. doi:10.1016/j.elspec.2013.11.006

15. Felicissimo, M. P.; Jarzab, D.; Gorgoi, M.; Forster, M.; Scherf, U.; Scharber, M. C.; Svensson, S.; Rudolf, P.; Loi, M. A. J. Mater. Chem. 2009, 19, 4899-4901. doi:10.1039/b906297a

16. Subías, G.; Abbaz, T.; Fabre, J. M.; Fraxedas, J. Phys. Rev. B 2007, 76, 085103. doi:10.1103/PhysRevB.76.085103

17. Fraxedas, J.; García-Gil, S.; Monturet, S.; Lorente, N.; Fernández-Torrente, I.; Franke, K. J.; Pascual, J. I.; Vollmer, A.; Blum, R.-P.; Koch, N.; Ordejón, P. J. Phys. Chem. C 2011, 115, 18640-18648. doi:10.1021/jp2050838

18. Albonetti, C.; Martínez, J.; Losilla, N. S.; Greco, P.; Cavallini, M.; Borgatti, F.; Montecchi, M.; Pasquali, L.; García, R.; Biscarini, F. Nanotechnology 2008, 19, 435303. doi:10.1088/0957-4484/19/43/435303

19. Way, W. K.; Rosencrance, S. W.; Winograd, N.; Shirley, D. A. Surf. Sci. Spectra 1993, 2, 67. doi:10.1116/1.1247712

20. Oria, L.; Ruiz De Luzuriaga, A.; Alduncin, J. A.; Pérez-Murano, F. Microelectron. Eng. 2013, 110, 234-240. doi:10.1016/j.mee.2012.12.006

21. Rosencrance, S. W.; Way, W. K.; Winograd, N.; Shirley, D. A. Surf. Sci. Spectra 1993, 2, 71. doi:10.1116/1.1247740

22. Varlot, K.; Martin, J. M.; Quet, C. J. Microsc. 1998, 191, 187-194. doi:10.1046/j.1365-2818.1998.00376.x

23. Massey, S.; Cloutier, P.; Bazin, M.; Sanche, L.; Roy, D. J. Appl. Polym. Sci. 2008, 108, 3163-3168. doi:10.1002/app.27892

24. Klauser, R.; Hong, I.-H.; Su, H.-J.; Chen, T. T.; Gwo, S.; Wang, S.-C.; Chuang, T. J.; Gritsenko, V. A. Appl. Phys. Lett. 2001, 79, 3143. doi:10.1063/1.1415415

25. Lazzarino, M.; Heun, S.; Ressel, B.; Prince, K. C.; Pingue, P.; Ascoli, C. Appl. Phys. Lett. 2002, 81, 2842. doi:10.1063/1.1509121

26. Tanuma, S.; Powell, C. J.; Penn, D. R. Surf. Interface Anal. 1991, 17, 911-926. doi:10.1002/sia.740171304 
27. Sparnacci, K.; Antonioli, D.; Gianotti, V.; Laus, M.; Ferrarese Lupi, F.; Giammaria, T. J.; Seguini, G.; Perego, M. ACS Appl. Mater. Interfaces 2015, 7, 10944-10951. doi:10.1021/acsami.5b02201

28. Sparnacci, K.; Antonioli, D.; Perego, M.; Giammaria, T. J.; Seguini, G.; Ferrarese Lupi, F.; Zuccheri, G.; Gianotti, V.; Laus, M. Polym. Int. 2017, 66, 459-467. doi:10.1002/pi.5285

29. Garcia, R.; Calleja, M.; Pérez-Murano, F. Appl. Phys. Lett. 1998, 72, 2295. doi:10.1063/1.121340

30. Schaefers, F.; Mertin, M.; Gorgoi, M. Rev. Sci. Instrum. 2007, 78, 123102. doi:10.1063/1.2808334

31. Gorgoi, M.; Svensson, S.; Schäfers, F.; Öhrwall, G.; Mertin, M.; Bressler, P.; Karis, O.; Siegbahn, H.; Sandell, A.; Rensmo, H.; Doherty, W.; Jung, C.; Braun, W.; Eberhardt, W. Nucl. Instrum. Methods Phys. Res., Sect. A 2009, 601, 48-53. doi:10.1016/j.nima.2008.12.244

32. Ton-That, C.; Shard, A. G.; Teare, D. O. H.; Bradley, R. H. Polymer 2001, 42, 1121-1129. doi:10.1016/S0032-3861(00)00448-1

33. Verdaguer, A.; Weis, C.; Oncins, G.; Ketteler, G.; Bluhm, H.; Salmeron, M. Langmuir 2007, 23, 9699-9703. doi:10.1021/la700893w

34. Himpsel, F. J.; Hollinger, G.; Pollak, R. A. Phys. Rev. B 1983, 28, 7014-7018. doi:10.1103/PhysRevB.28.7014

35. Takata, Y.; Kayanuma, Y.; Yabashi, M.; Tamasaku, K.; Nishino, Y.; Miwa, D.; Harada, Y.; Horiba, K.; Shin, S.; Tanaka, S.; Ikenaga, E.; Kobayashi, K.; Senba, Y.; Ohashi, H.; Ishikawa, T. Phys. Rev. B 2007, 75, 233404. doi:10.1103/PhysRevB.75.233404

36. Kövér, L. J. Electron Spectrosc. Relat. Phenom. 2010, 178-179, 241-257. doi:10.1016/j.elspec.2009.12.004

37. Wippler, D.; Wilks, R. G.; Pieters, B. E.; van Albada, S. J.; Gerlach, D.; Hüpkes, J.; Bär, M.; Rau, U. ACS Appl. Mater. Interfaces 2016, 8, 17685-17693. doi:10.1021/acsami.6b04666

\section{License and Terms}

This is an Open Access article under the terms of the Creative Commons Attribution License (http://creativecommons.org/licenses/by/4.0), which permits unrestricted use, distribution, and reproduction in any medium, provided the original work is properly cited.

The license is subject to the Beilstein Journal of Nanotechnology terms and conditions: (http://www.beilstein-journals.org/bjnano)

The definitive version of this article is the electronic one which can be found at: doi:10.3762/bjnano.8.198 